\title{
Raman opalescence and central peak scattering near phase transition point in crystals
}

\author{
V.S. Gorelik ${ }^{1,2}$ \\ ${ }^{1}$ P.N. Lebedev Physical Institute of the Russian Academy of Sciences, 119991, Moscow, Russia \\ ${ }^{2}$ Bauman Moscow State Technical University, 105005, Moscow, Russia \\ gorelik@sci.lebedev.ru
}

The investigations of the peculiarities in light scattering near the phase transitions in media are important direction of the modern physics. Because of the strong fluctuations increase near the phase transition point, the opalescence effect, i.e. the sharp growth of light scattering intensity, has been observed. At the beginning of the twentieth century Smolukhovsky, Keesom, Ornstein and Zernike have investigated opalescence near phase transition points of liquids. Later A. Yakovlev, L.M. Mikheeva, and T.S. Velichkina [1,2] have observed the opalescence in crystalline quartz within a narrow temperature interval of the $\alpha-\beta$ phase transition $\left(T_{C}=846 \mathrm{~K}\right)$.

Besides, so-called central peak, corresponding to a sharp spectral intensity increase in light, slow neutron, and $\mathrm{X}$-ray scattering in the region of structural phase transitions, was detected in several crystals: $\mathrm{Nb}_{3} \mathrm{Sn}, \mathrm{SrTiO}_{3}, \mathrm{KMnF}_{3}, \mathrm{KNbO}_{3}, \mathrm{~Pb}_{5} \mathrm{Ge}_{3} \mathrm{O}_{11}$ and others. Today numerous works [3-8] are devoted to the investigations of light scattering laws near the phase transition points in condensed media. In this paper, we present an experimental and theoretical investigation of Raman scattering intensity sharp increase, named as Raman opalescence, for some crystals near the phase transition point.

We have used the new experimental technique for Raman opalescence revealing, based on analysis of so called isofrequency temperature dependencies at low frequency spectral region. We have observed the sharp increase of the isofrequency peak spectral Raman intensity at low frequency Raman shift if the crystal temperature was approaching to the phase transition point. The experimental results are presented for different crystals, including ferroelectrics $\left(\mathrm{LiTaO}_{3}\right.$, $\left.\mathrm{LiNbO}_{3}, \mathrm{~Pb}_{5} \mathrm{Ge}_{3} \mathrm{O}_{11}, \mathrm{Ba}_{2} \mathrm{NaNb}_{5} \mathrm{O}_{15}\right)$ and ferroics $\left(\mathrm{SiO}_{2}, \mathrm{SrTiO}_{3}\right)$. Central peak scattering has been observed in isofrequency temperature light scattering dependencies at the vicinity of phase transition point.

The theory of central peak scattering, based on the strong interaction between soft mode and low frequency, good quality, nonfundamental oscillator has been developed. The effect of Hyper Raman opalescence as sharp increase of light scattering at the spectral range of the second harmonic generation near the phase transition point in crystals is described.

This work was supported in part by RFBR grant 18-02-00181.

1. I.A. Yakovlev, L.M. Mikheeva, T.S. Velichkina, Reports of the Academy of Sciences of the USSR 107, 675 (1956).

2. I.A. Yakovlev, L.M. Mikheeva, T.S. Velichkina, Kristallografiya 1, 123 (1956).

3. A.A. Anikev, V.S. Gorelik, B.S. Umarov, Sov. Phys. Sol. State 26, 1679 (1984).

4. V.S. Gorelik, P.P. Sverbil, A.I. Vodchits, et al., Bulletin of the Russian Academy of Sciences: Physics 82, 57 (2018).

5. V.S. Gorelik, A.Yu. Pyatyshev, Bulletin of the Russian Academy of Sciences: Physics 82, 299 (2018).

6. A.A. Anikiev, M.P. Umarov, Optics and Spectroscopy 125, 22 (2018).

7. A.A. Anikev, M.P. Umarov, J.F. Scott, ATP Advances 8, 115016 (2018).

8. N. Lagakos, H.Z. Cummins, Physical Review B 10, 1063 (1974). 